\title{
Commentary on: Reoperation rates for pelvic organ prolapse repairs with biologic and synthetic grafts in a large population-based cohort
}

\author{
Radhika Patnam ${ }^{1}$
}

Received: 12 July 2019 / Accepted: 5 September 2019/Published online: 31 October 2019

(C) The International Urogynecological Association 2019

The purpose of this study was to use database information to compare synthetic to biologic grafts for pelvic organ prolapse repairs. The recent FDA ban of mesh for pelvic organ prolapse repairs suggests that other options including biologic grafts need to be considered. While short-term follow-up has shown an increase in recurrence in biologic grafts and mesh complications in synthetic grafts, there are no data on longer term outcomes.

Using the California Office of Statewide Health Planning and Development database, they identified 14,192 women who had graft for POP repair between 2005 and 2011, with $14 \%$ having a biologic graft and $86 \%$ having a synthetic graft. The median follow-up was 745 days, and 788 (5.6\%) women had repeat surgery. Biologic grafts had significantly more reoperations from POP recurrence (3.6\% vs. $2.5 \%, p=0.01)$, whereas synthetic grafts had significantly more reoperations from mesh complications ( 3.0 vs. $2.0 \%, p=0.02)$. These trends were maintained over time. With multivariable modeling, there was no difference in overall risk of all-cause surgery.
They also found that White race, younger age and hypertension were associated with an increased rate of all-cause reoperation. Obesity, hypertension and younger age were also associated with higher odds for reoperation secondary to graft complication.

Discussing other methods for POP repair in this study is helpful after the recent FDA ban. While biologic grafts were used significantly less in this population, that may change with the lack of other options beyond native tissue available for POP repair. Understanding outcomes in the longer term period, here 2 years, allows for providers to offer data to allow patients to make informed decisions. Unfortunately, this study only encompasses those who opted for reoperation, leaving > $90 \%$ of those who underwent a graft procedure unaccounted. Given this, while this study adds to the growing body of evidence about grafts, further studies must be done to endorse more common use of biologic grafts, especially as providers and patients look to other avenues with the removal of vaginal synthetic mesh for pelvic organ prolapse repair.
Radhika Patnam

radhika_patnam@med.unc.edu

1 University of North Carolina at Chapel Hill, Chapel Hill, NC, USA 